\title{
A Socioeconomic Well-Being Index
}

\author{
A. Alexandre Trindade ${ }^{1}$, Abootaleb Shirvani ${ }^{1}$, Xiaohan $\mathrm{Ma}^{2}$ \\ ${ }^{1}$ Texas Tech University, Department of Mathematics \& Statistics, Lubbock TX 79409-1042, U.S.A. \\ ${ }^{2}$ Texas Tech University, Department of Economics, Lubbock TX 79409-1014, U.S.A. \\ Correspondence: Abootaleb Shirvani, Texas Tech University, Department of Mathematics \& Statistics, Lubbock TX \\ 79409-1042, U.S.A.
}

Received: March 27, 2020

Accepted: May 11, $2020 \quad$ Available online: May 15, 2020

doi:10.11114/aef.v7i4.4855

URL: https://doi.org/10.11114/aef.v7i4.4855

\begin{abstract}
An annual well-being index constructed from thirteen socioeconomic factors is proposed in order to dynamically measure the mood of the US citizenry. Econometric models are fitted to the log-returns of the index in order to quantify its tail risk and perform option pricing and risk budgeting. By providing a statistically sound assessment of socioeconomic contentment, the index is consistent with rational finance theory, enabling the construction and valuation of insurance-type financial instruments to serve as contracts written against it. Endogenously, the VXO volatility measure of the stock market appears to be the greatest contributor to tail risk. Exogenously, "stress-testing" the index against the politically important factors of trade imbalance and legal immigration, quantify the systemic risk. For probability levels in the range of $5 \%$ to $10 \%$, values of trade below these thresholds are associated with larger downward movements of the index than for immigration at the same level. The main intent of the index is to serve as an early-warning mechanism for negative changes in the mood of citizens, thus alerting policy makers and private agents to potential future market downturns.
\end{abstract}

Keywords: Econometrics, option pricing, risk budgeting, stress-testing

\section{Introduction}

It is an obvious statement that financial market participants dislike disruptions, especially those that are not based on economic fundamentals. If an economy is likely to enter a recession, fully informed and rational investors would store their wealth in more safe assets, or search for alternative investment opportunities. Although the trough of a recession tends to materialize slowly enough to give investors time to prepare for it, opportunist or uninformed traders, by contrast, may decide to ride the bubble until the very end. For example, there were signs of the crash of 2008 up to one year in advance, however, the overwhelming majority of market participants were content in simply riding the bubble in 2007 and early 2008. The tragedy of most investors then was that they decided to take their positions only months ahead of the crash, resulting in a "crowding effect" where all investors "rushed out of the door", like in a bank run, thus creating a market avalanche. A consequence of this is that market crowding becomes an increasingly popular research topic. Although the reasons for it are now well known, few had any idea about its potential severity in 2007 before the Great Recession.

Nowadays, potential sharp market downturns are studied very carefully. Regulators instituted very strict standards with the Basel III Accord, and the capital requirements for firms that are "too big to fail" are now quite severe. Even in high frequency trading, flash-crashes are becoming rare. However, financial markets may still experience disruptions, due to, for example, an increase in uncertainty associated with geopolitical events or disease outbreaks (e.g., the current coronavirus pandemic). Moreover, policy makers themselves may be as uninformed as private agents, and typically do not have perfect information on the state of the economy. Policy decisions thus made may not be effective in stabilizing the financial market, or in serving as a useful signal for private agents. ${ }^{1}$ Finally, the mood (sentiment) of economic agents, as exemplified by the crowding effect discussed earlier, may play a crucial rule in the stabilization of financial markets and the macroeconomy.

\footnotetext{
${ }^{1}$ For example, the current yield curve is "bumpy" suggesting that markets may be uncertain about future monetary policy.
} 
The objective of this research is to employ state-of-the-art statistically sound financial methods to construct a (to the best of our knowledge) reliable and dynamic aggregate index based on a variety of macro and micro economic factors, which will provide a quantitative snapshot assessment of the US citizenry's level of socioeconomic content. ${ }^{2}$ We term our proposed index the Socioeconomic WellBeing Index (SWBI). ${ }^{3}$ While also "stress-testing" against potential external factors like immigration and trade imbalance, the index aims to determine the level of future systemic risk, thereby serving as an "early-warning" mechanism for serious potential undercurrent issues that could precipitate from changes in the mood of citizens. In turn, this leads to potential future crises that may be even more severe than in 2008 . The SWBI assesses the tail risk (due to extreme events) and provides forward-looking distributions for economic risk factors and social well-being downturns.

Specifically, the SWBI does this by being based on the log-returns of an equally-weighted linear combination of factors. Econometric ARMA-GARCH models driven by generalized hyperbolic noise, satisfactorily capture the serial dependence and estimate the cross-sectional distribution of the data, as predicted by contemporary best-practices financial theory (Massing, 2019). This framework also allows for the generation of Monte Carlo based future price scenarios, leading to option pricing and risk budgeting for the SWBI. This enables the construction and valuation of insurance-type financial instruments. Among the component series of the SWBI, the VXO volatility measure of the stock market is the greatest contributor to tail risk. Completing the rational finance-based valuation, stress-testing of the SWBI against external factors like trade imbalance and amount of legal immigration, quantifies the level of systemic risk. In this regard we find that the level of trade imbalance tends to be associated with a larger impact on negative well-being than does immigration.

There have been several recent noteworthy attempts by academics and non-academics alike to quantify the well-being of the nation, and/or to explore its implications on economic performance. Most studies approach this issue via surveys, thereby producing subjective indicators of contentment. For example, the Gallup-Sharecare Well-Being Index is constructed from monthly telephone interviews on how people perceive and experience their daily lives through five perspectives: purpose, social, financial, community, and physical. The Gallup-Healthways Well-Being Index interviews 1,000 US adults daily to provide real-time measurement of health and well-being. The World Happiness Report combines various global household surveys to construct three main happiness measures: life evaluations, positive effect, and negative effect. The National Accounts of Time Use and Well-being is used by Krueger (2009), among others, to construct the subjective well-being of nations.

The index we propose here differs from the above measures by being based on time series of important macroeconomic aggregates, which would arguably be more comprehensive, as well as immune from possible response bias associated with subjective surveys ${ }^{4}$. A strand of the literature has already focused on such socioeconomic factors affecting people's well-being. Blanchflower and Oswald (2004) documents happiness trends in the US and Great Britain, and quantitatively estimates the dollar values of events like unemployment and divorce as sources influencing happiness. Ferrer-i Carbonell and Frijters (2004) develops a conditional estimator for the fixed-effect ordered logit model to re-evaluate the micro-level determinants of happiness. Di Tella et al. (2003) shows that macroeconomic movements, such as gross domestic product and unemployment benefits, have significant impact on national well-being.

The main contribution of our paper, however, is in constructing a national well-being index based on financial econometric modeling and dynamic asset pricing theory. The importance of this well-being barometer is understood in light of recent geopolitical events, such as the yellow-vests movement in France, and the Hong-Kong protests. It seems that a reasonable predictor of revolt and instability in developed and industrialized nations is the magnitude of not only discontent, but also contentment, above/below some historical average level. These observations behoove us to concentrate on the downside risk and volatility, rather than on the direction of the index, and is the primary reason for our devoting careful attention to tail risk measures. Our modeling can ultimately be used for scenario forecasting and the issuing of marketable financial contracts, such as options and futures.

The rest of the paper is structured as follows. Section 2 describes the set of socioeconomic factors to be used to quantify the index, the construction of which is detailed in Section 3. This is followed by econometric time series modeling of the index and its marginal density estimation in Section 4, this being a prerequisite step for the option pricing and risk

\footnotetext{
${ }^{2}$ We do not employ behavioral finance as it is not consistent with rational finance, and is thus unable to aid in the construction of insurance-type financial instruments to serve as (financial) contracts written against the index.

${ }^{3}$ In contrast to many existing indices, the SWBI includes not only economic, but also diverse data related to social well-being.

${ }^{4}$ See McLean (2014) for a survey of national and international indices of well-being.
} 
budgeting steps in Sections 5 and 6, respectively. The paper ends with a stress-testing analysis in Section 7, where the effect of external adverse socioeconomic factors on the tail risk is examined. A discussion rounds out the paper.

\section{Data Description}

The variables we select are those that have been shown in the literature to affect the well-being of economic agents, such as in Krueger (2009), Blanchflower and Oswald (2004), Di Tella et al. (2003), Ferrer-i Carbonell and Frijters (2004), among others. Abbreviated names for our list of 13 factors as well as their precise description is as follows. (We append the prefix Neg to some of these if we wish to also consider the reversed-sign version.)

Confidence. The Consumer Confidence Index provides an indication of future developments of household consumption and savings, calculated based upon answers regarding their expected financial situation, their sentiment about the general economic situation, unemployment, and capability of savings.

CPI. Inflation as measured by consumer price index (CPI), is the growth rate of CPI for all urban consumers, which is a measure of the average change rate in the price for goods and services paid by urban consumers between any two time periods. (Reversed-sign version: NegCPI.)

CrimeRate. Crime rate represents the estimated amounts of violent crimes per 100,000 people. (Reversed-sign version: NegCrimeRate.)

DispIncome. Disposable income represents real disposable personal income, which is inflation adjusted personal income after payment of taxes.

GDP. Real GDP (Gross Domestic Product) is the inflation adjusted value of the final goods and services produced by labor and property located in the United States.

GenderParity. Gender parity is an index measuring the relative access to primary and secondary education for males and females, calculated as the quotient of the number of females to the number of males enrolled in the given stage of education. (Reversed-sign version: NegGenderParity.)

GovTrans. Government transfer is the amount of government social benefits provided to the unemployed, also known as unemployment insurance.

Inequality. Inequality is the Gini index of income inequality, measuring household income dispersion. (Reversed-sign version: NegInequality.)

LifeExpect. Life expectancy indicates the number of years a newborn infant would live if prevailing patterns of mortality at the time of its birth were to stay the same throughout its life.

Sentiment. The index of Consumer Sentiment is an economic indicator that measures how optimistic consumers perceive about their financial conditions and the state of the economy, constructed based on survey questions in the Survey of Consumers.

Uncertainty. Uncertainty indicates the US policy uncertainty index based on newspaper coverage frequency, the increase of which is found to foreshadow declines in investment, output, and employment in the United States. (Reversed-sign version: NegUncertainty.)

Unemploy. Unemployment represents the number of unemployed as a percentage of the labor force (people 16 years of age and older, who currently do not reside in institutions, and who are not on active duty in the Armed Forces). (Reversed-sign version: NegUnemploy.)

VXO. The VXO index is the CBOE S\&P 500 Volatility Index, calculated by the Chicago Board Options Exchange (CBOE), and measuring the overall short-term volatility in the stock market. (Reversed-sign version: NegVXO.)

Of these, real GDP, inflation, unemployment, government transfer, life expectancy, disposable income, and VXO, are obtained from the data set of the Federal Reserve Bank of St. Louis. Inequality is obtained from the US Census Bureau. Crime rate is calculated by the FBI. The uncertainty index is developed by Baker et al. (2016). Consumer confidence is provided by OECD (Organization for Economic Co-operation and Development) in its publication of Main Economic Indicators: Business tendency and consumer opinion surveys. Gender parity index is obtained from UNESCO (The United Nations Educational, Scientific and Cultural Organization).

The common period of these 13 yearly factors is 1986-2016. Figures 1a and 1b display time series of their actual values. They seem to be of two distinct types: GDP, CPI, Inequality, LifeExpect, CrimeRate, and DispIncome display trending behavior, while the remaining 7 appear to be stationary. (However, at the 5\% level of significance the Augmented Dickey-Fuller test does not reject the null hypothesis of unit-root nonstationarity for any of the series.) 


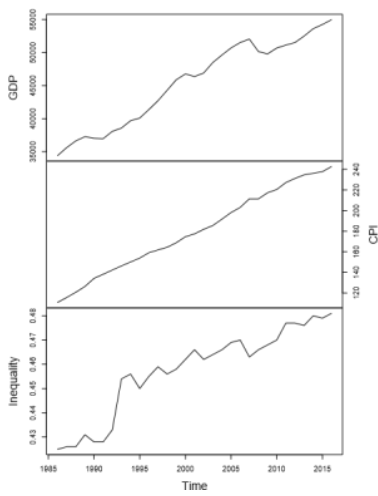

(a) Trending.

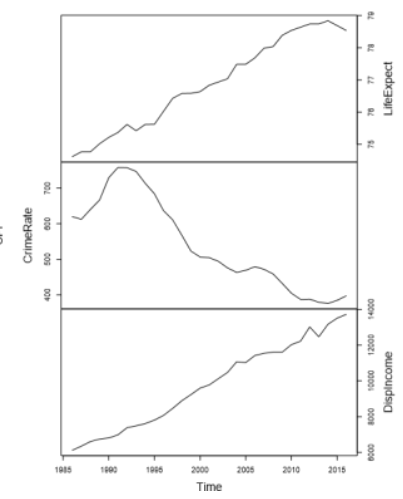

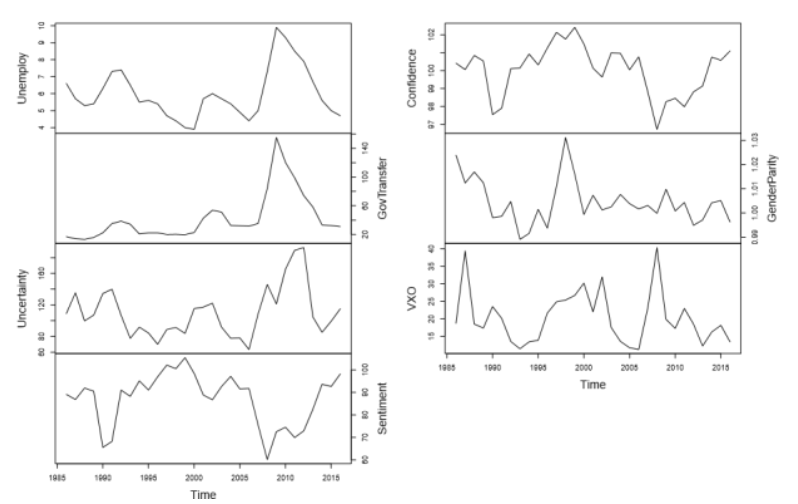

(b) Stationary.

Figure 1. Time series of actual values for the factors with trending and with stationary behavior

At this point we examined the existence of cointegration relationships among the series in each of the two groups. For the trending group, the strong trends coupled with small sample size does not allow for estimation of an underlying VAR model necessary for carrying out Johansen's cointegration test (Johansen, 1988). For the stationary group, we find 2 cointegrating relationships at the $5 \%$ level of significance based on a VAR(2). (Higher order VAR models could not be fitted due to the similar issue of collinearity and small sample size.) Figure 2 displays cross-sectional scatterplots of actual values for all 13 factors. We note that the large degree of collinearity is easily spotted from the correlations on the upper triangular portion, which are displayed with font size proportional to the magnitude. There is therefore the question of whether all series are necessary for the construction of the SWBI; an issue to be explored in the next section.

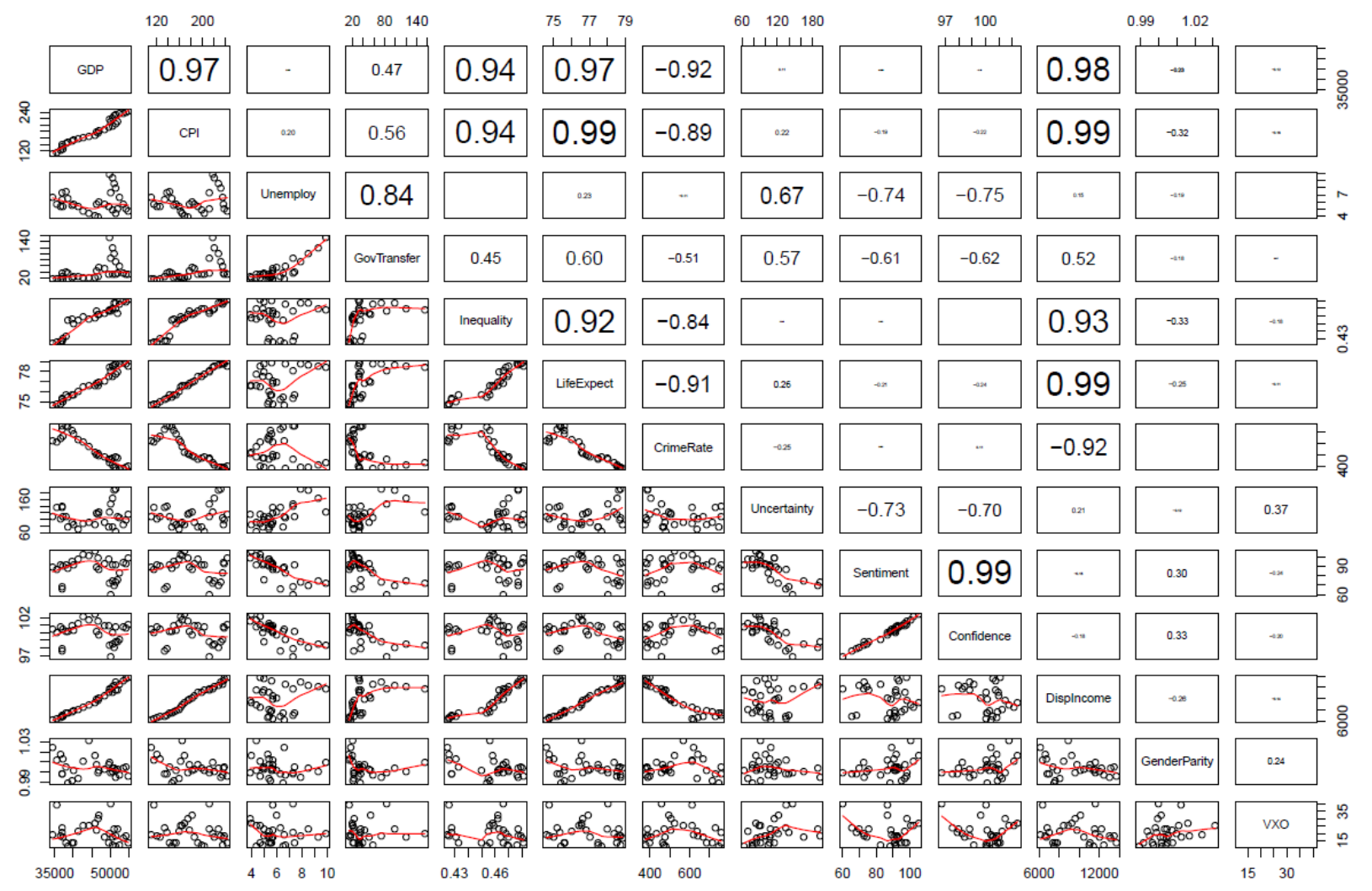

Figure 2. Cross-sectional scatterplots of actual values for the 13 factors 


\section{Construction of the Index}

In this section we detail the methodology for constructing the SWBI. It employs the 13 factors discussed in the previous section. As is typical of macroeconomic variables, the Augmented Dickey-Fuller Test gives large p-values for all series, confirming that they are all $I(1)$, or integrated of order one. Thus, and in order to arrive at plausibly stationary series, we transformed the actual values to $\log$-returns, i.e., if $P(i, t)$ denotes the value of the $i$-th factor at time $t$, then $r(i, t)=\log$ $P(i, t)-\log P(i, t-1)$ denotes its log-return.

In order to have positive values equate to greater well-being, we reversed the sign of the log-return value associated with the following factors: CPI, Unemploy, Inequality, CrimeRate, Uncertainty, GenderParity, and VXO. On their original scale, it can be argued that large values of these factors would tend to be associated with decreased well-being. We further set the 1986 return value to zero for all factors, so that our starting point is the panel of log-returns:

$$
\{r(i, t)\}, \quad i=1, \ldots, N=13, \quad t=1, \ldots, T=30 .
$$

Time series plots of $r(i, t)$ for each of $i$ (each factor) are displayed in Figure 3a. For comparison, the group whose sign was reversed is shown in a different color and line type. All series appear to be stationary now; at least with respect to trends and cycles.

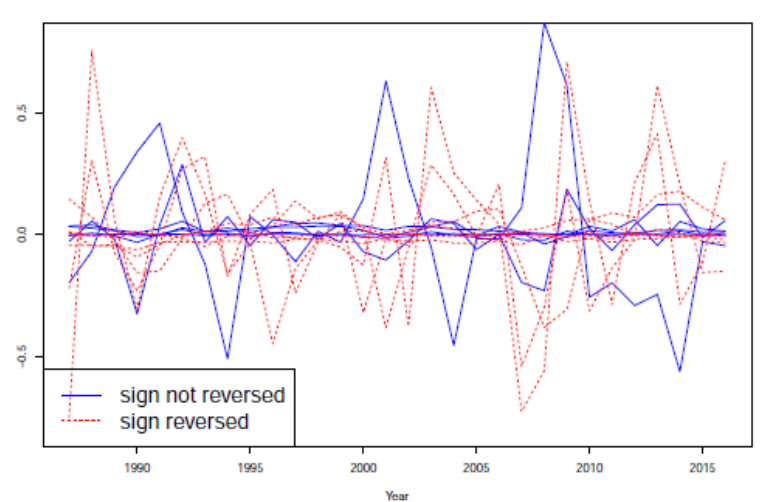

(a)

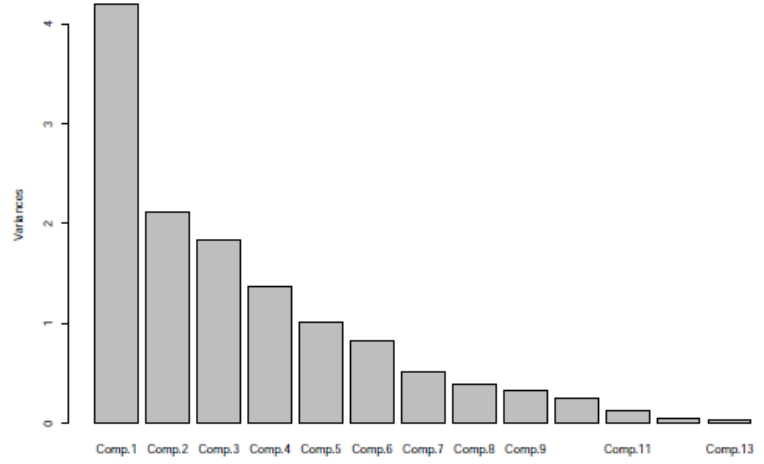

(b)

Figure 3. Analysis for the panel of log-returns, $r(i, t)$, defined in (1). (a) Time series plots with color and line type coded according to whether the sign was reversed (dotted) or not (solid). (b) Scree plot from a PCA giving the proportion of contribution from each component toward explaining the 13-dimensional correlation matrix

The formation of the SWBI value at time $t, r_{t}$, is now obtained as an equally weighted linear combination of the factors in the panel of $r(i, t)$ values. This is done after first standardizing each factor series, so as to extract out the location-scale effects. The (averaged over all factors) location and scale are then added back in. Specifically, we implement the following algorithm.

Algorithm 1 (Formation of SWBI) Starting from the panel of time series $r(i, t)$ in (1), proceed as follows:

(i) For each $i=1,2, \ldots . . N$, standardize $r(i, t)$ according to its factor-level mean and standard deviation:

$$
R(i, t)=\frac{r(i, t)-m(i)}{s(i)}, \quad \quad m(i)=\frac{1}{T} \sum_{t=1}^{T} r(i, t), \quad s(i)=\frac{1}{T-1} \sum_{t=1}^{T}[r(i, t)-m(i)]^{2} .
$$

(ii) Form the standardized index return series by weighting equally across all factors: 


$$
R(t)=\frac{1}{\sqrt{N}} \sum_{i=1}^{N} R(i, t)
$$

(iii) Form the annual index return series by undoing the standardization in (i) according to the average of the factor means and standard deviations:

$$
r_{t}=m+s R(t), \quad m=\frac{1}{N} \sum_{i=1}^{N} m(i), \quad s=\frac{1}{N} \sum_{i=1}^{N} s(i) .
$$

For ease of reference in subsequent analyses, we call the resulting $r_{t}$ series simply as the Index or SWBI. A time series plot is displayed in the top left panel of Figure 4.

At this point, and with plausibly stationary factor log-returns as gleaned from Figure 3a, one can properly investigate the issue of whether all 13 factors that make up $r_{t}$ are needed. A principal component analysis (PCA) on these series suggests we need almost all factors (correlation matrix based). Although the 1st PCA explains $32 \%$ of the variability, there is a very gradual contribution from each additional component, so that it is not until the 9th PCA that we obtain an explanatory capability from these factors exceeding $95 \%$. Moreover, the weights in each PCA are spread over almost all factors in each of these first 9 PCAs. In summary, there is not an overwhelmingly clear indication that we should reduce the dimensionality of the vector of 13 factors.

\section{Econometric Analysis and Forecasting}

In this section we fit time series models to $r_{t}$. This will allow for evaluation of risk measures and the performing of option pricing for the SWBI. Two models were entertained here. Searching for the best-fitting ARIMA model via AIC and BIC points to an ARIMA $(0,0,0)$ with zero mean, i.e., white noise. However, since the option pricing relies on an ARMA-GARCH configuration, we fit also an $\operatorname{ARMA}(1,1)-\operatorname{GARCH}(1,1)$ with zero mean and normal innovations. The innovations from this fit (the raw residuals divided by the GARCH conditional standard deviation estimate) are displayed in the top right panel of Figure 4.

The generation of multiple scenarios from the (stationary distribution of the) fitted model for $r_{t}$ in order to calculate risk measures, a step we loosely call "forecasting", can be performed once an appropriate model for the innovations has been determined. The standard parametric family in this context is the Generalized Hyperbolic (GH) distribution, originally introduced by Barndorff-Nielsen (1977). Jorgensen (1982) is the classical reference on its properties, while an accessible overview is given by Paolella (2007). Briefly, the GH features both heavy tails and skewness, includes the familiar elliptical family as a special case (normal, t, etc.), exhibits tail-dependence (Schmidt, 2007), and is infinitely divisible, a necessary and sufficient condition to build Levy processes (ubiquitous in financial time-series due to their continuity and ability to model jumps). For these and other reasons it has become the default distribution in modeling the returns of equity indices at various temporal scales (Massing, 2019).

Notationally, we designate by $X \sim \mathrm{GH}(\lambda, \alpha, \beta, \delta, \mu)$ a random variable following a GH with parameters $\lambda \in \mathrm{R}$ (tail heaviness), $\alpha>0$ (shape), $\beta \in \mathrm{R}$ (skewness) such that $\alpha^{2}-\beta^{2}>0, \delta>0$ (scale), and $\mu \in \mathrm{R}$ (location). (There are at least two alternative parametrizations in common usage). The density function is derived by mixing normals according to a Generalized Inverse Gaussian distribution (a special case of $\mathrm{GH}$ ), and does not therefore in general have a closed-form representation. Software implementation of the GH is provided through the R library ghyp (Luethi and Breymann, 2016) and its accompanying vignette. Two special cases of the GH we focus on in this study are the Variance Gamma (VG), which is a GH with $\lambda>0$ and $\delta=0$, and the Negative Inverse Gaussian (NIG), a GH with $\lambda=-1 / 2$; both of these possessing exponential tails. The latter in particular is pointed out by Barndorff-Nielsen (1997) and Barndorff-Nielsen (2007) as being especially appropriate for stochastic volatility modeling based on a homogeneous Levy process, and the option pricing models that can consequently be constructed. 
The Index r(t)

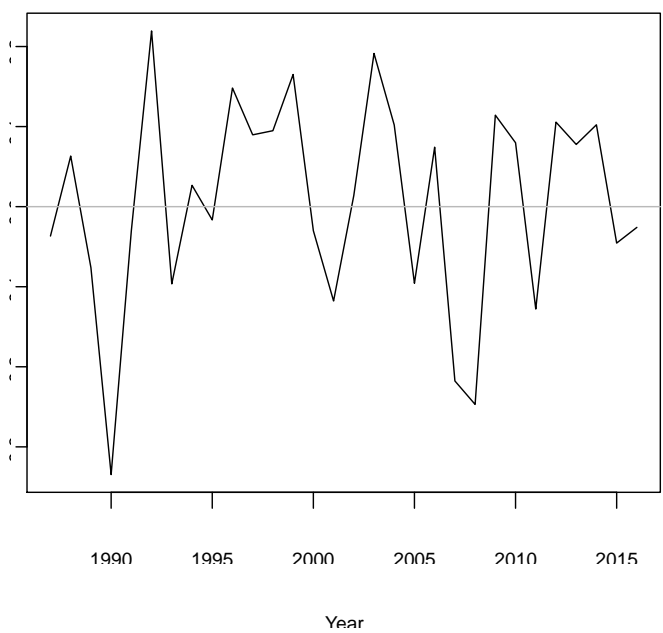

Estimated density of innovations

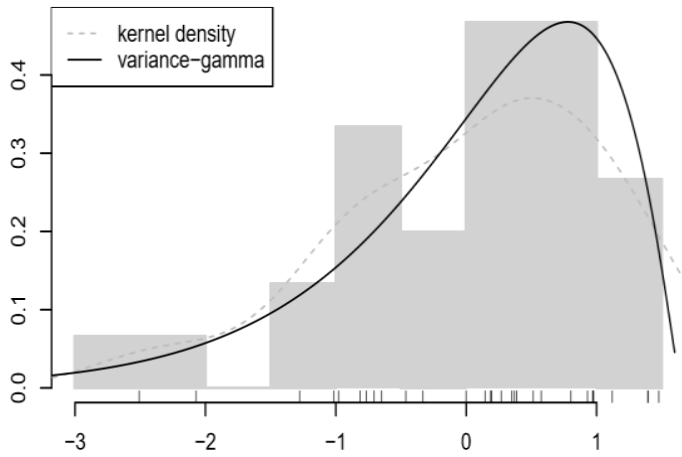

Innovations from ARMA-GARCH fit to $\mathbf{r}(\mathrm{t})$

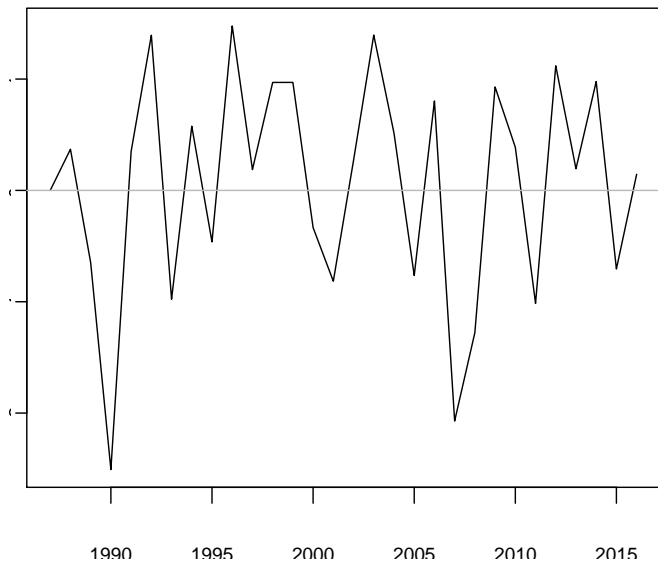

Time

Simulated density of $\mathbf{r}(t)$ with VG innovations

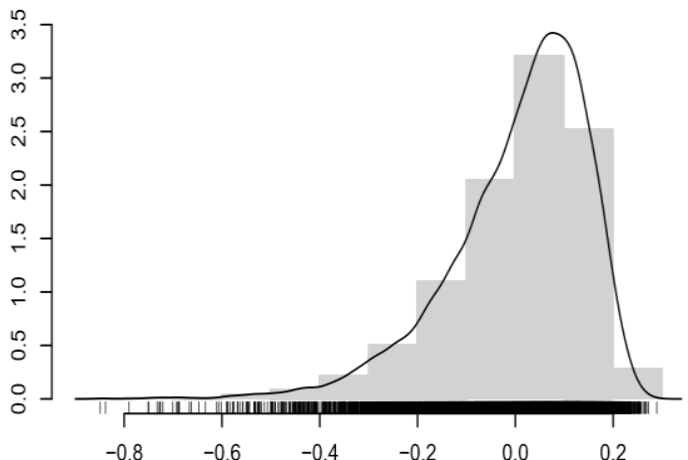

Figure 4. Time series of the Index $r_{t}$ and its innovations from an ARMA-GARCH fit (top panels). The bottom right panel displays 10,000 scenarios from the marginal of $r_{t}$ based on a VG distribution fitted to the ARMA-GARCH innovations (bottom left panel)

Fitting VG and NIG marginal models to the sample of $\operatorname{ARMA}(1,1)-\operatorname{GARCH}(1,1)$ innovations seen as the rug and histogram plot in the lower left panel of Figure 4, leads to the VG density displayed as the solid line in that figure (the NIG being an inferior fit). The appropriateness of the fit is confirmed by the Kolmogorov-Smirnov and Anderson-Darling tests with p-values exceeding 0.9. Simulating 10,000 scenarios from the overall fitted ARMA(1,1)-GARCH(1,1) model with VG innovations, then leads to the marginal of $r_{t}$ seen as the rug and histogram in the lower right panel of Figure 4; the solid line being a kernel density smoother. Summary statistics and left tail risk measures for these scenarios are shown on Table 1. Here, the Value-at-Risk (VaR) and expected shortfall (ES) risk measures are reported (Pflug and Werner, 2007, Ch. 2).

\section{Option Pricing}

Options can be used for hedging, speculating, and calculating the risk of investments. The most common option pricing models are Black-Scholes, Binomial, Trinomial tree, Monte-Carlo simulation, and finite difference. Recently, the discrete stochastic volatility-based model has received 
Table 1. Summary statistics and left tail risk measures for the 10,000 scenarios generated from the $\operatorname{ARMA}(1,1)-G A R C H(1,1)$ model with VG innovations fitted to the Index

Summary Statistics

\begin{tabular}{ccc} 
Minimum/Maximum & Mean/Median & Skewness/Kurtosis (excess) \\
\hline$-0.8492 / 0.2894$ & $0.0064 / 0.0351$ & $-1.181 / 2.094$ \\
\hline & Left Tail Risk Measures \\
$1 \% \mathrm{VaR} / \mathrm{ES}$ & $5 \% \mathrm{VaR} / \mathrm{ES}$ & $10 \% \mathrm{VaR} / \mathrm{ES}$ \\
\hline$-0.4411 /-0.5458$ & $-0.2655 /-0.3720$ & $-0.1826 /-0.2960$
\end{tabular}

considerable attention, particularly with regard to explaining some well-known mispricing phenomena. The pricing model in a discrete-time conditional heteroskedasticity setting was first considered by Duan (1995), who used a GARCH driven by normal innovations for asset returns in order to price options. We follow this strategy by considering a standard GARCH model with GH innovations to calculate $I_{t}$, the fair value of an option of SWBI at time $t$. Specifically, we assume the log-returns $r_{t}$ follow the process:

$$
r_{t}=\log \frac{I_{t}}{I_{t}-1}=r_{t}^{\prime}+\lambda_{0} \sqrt{h_{t}}-\frac{1}{2} h_{t}+\sqrt{h_{t}} \epsilon_{t}
$$

where $h_{t}=\mathrm{V}\left(r_{t} \mid F_{t-1}\right)$ is the conditional variance at time $t$, with $F_{t-1}$ denoting the information set consisting of all linear functions of past returns available up to time $t-1, r_{t}^{\prime}$ is the risk-less rate of return at time $t$, and $\lambda_{0}$ is the risk premium for the SWBI. We use a GARCH(1,1) with GH innovations to model the conditional variance as follows:

$$
h_{t}=m+a h_{t-1}+b \epsilon_{t-1}^{2}, \quad\left\{\epsilon_{t}\right\} \sim i i d \mathrm{gH}(\lambda, \alpha, \beta, \delta, \mu)
$$

Blaesild (1981) proved that under this model, the conditional distribution of $r_{t}$ given $F_{t-1}$ on the real-world probability space $\mathrm{P}$ is distributed as

$$
r_{t} \sim\left(\lambda, \frac{\alpha}{\sqrt{h_{t}}}, \frac{\beta}{\sqrt{h_{t}}}, \delta \sqrt{h_{t}}, r_{t}^{\prime}+m_{t}+\mu \sqrt{h_{t}}\right), \quad \text { with } \quad m_{t}=\lambda_{0} \sqrt{h_{t}}-\frac{1}{2} h_{t}
$$

Options are priced on the risk-neutral probability (probability space of future outcomes adjusted for risk). In an incomplete market to price options, the crucial issue is to identify an equivalent martingale measure to obtain a consistent price for contingent claim. Using the Gerber and Shiu (1994) Esscher transformation, Chorro (2012) proved that, under model (3), the conditional distribution of $r_{t}$ given $F_{t-1}$ on the risk-neutral probability space $\mathrm{Q}$ is distributed as

$$
r_{t} \sim\left(\lambda, \frac{\alpha}{\sqrt{h_{t}}}, \frac{\beta}{\sqrt{h_{t}}}+\theta_{t}, \delta \sqrt{h_{t}}, r_{t}^{\prime}+m_{t}+\mu \sqrt{h_{t}}\right)
$$

where, and with $M($.$) denoting the conditional moment generating function of r_{t+1}$ given $F_{t}$ on $P, \theta_{t}$ solves the equation

$$
M\left(1+\theta_{t}\right)=M\left(\theta_{t}\right) \exp \left\{r_{t}^{\prime}\right\}
$$


(See Duffie (2001) for details on the precise definitions of the probability spaces P and Q.)

To price the SWBI call option up to a given time to maturity $T$, we use Monte Carlo simulation to generate future values of SWBI via the scheme implemented by Chorro (2012), as follows:

1. Fit the GARCH $(1,1)$ model (3) to the available historical log-returns of SWBI.

2. Set $t=0$ and forecast $h_{1}$, the conditional variance at time $t=1$.

3. Starting from $t=1$, repeat steps (a)-(c) below under $\mathrm{Q}$ for $t=1, \ldots, T$ :

(a) solve (6) to find $\theta_{t}$;

(b) generate $\epsilon_{t}$ from the stationary distribution of $\epsilon_{t} \sim\left(\lambda, \alpha, \beta+\sqrt{h_{t}} \theta_{t}, \delta, \mu\right)$;

(c) compute $r_{t+1}$ and $h_{t+1}$.

4. This scheme yields $\left\{r_{1}, \ldots, r_{T}\right\}$ under $Q$. Thus, the future price of the SWBI at time $T$ is given by

$$
I_{T}=I_{0} \exp \left\{\sum_{t=1}^{T} r_{t}\right\}
$$

5. Repeat step (3)- (4) in order to simulate $N=10,000$ future price values of the SWBI:

$$
\left\{I_{T}^{(1)}, \ldots, I_{T}^{(N)}\right\} .
$$

The approximate call $(C)$ and put $(P)$ option prices at time $t \leq T$ are then computed, for a given strike price $K$, as the Monte Carlo averages:

$$
\begin{aligned}
& C(t, T, K)=e^{-r_{t}^{\prime}(T-t)} \frac{1}{N} \sum_{i=1}^{N}\left(I_{T}^{(i)}-K\right)_{+} \\
& P(t, T, K)=e^{-r_{t}^{\prime}(T-t)} \frac{1}{N} \sum_{i=1}^{N}\left(K-I_{T}^{(i)}\right)_{+} .
\end{aligned}
$$

Figures 5-7 show call option prices, put option prices, and call-put option prices, respectively, plotted against both maturity $(T)$ and strike $(K)$. The graphs reveal the relationships among time to maturity, strike, and option prices. As expected, for a fixed $K$ we see a decline in price as $T$ increases. Figure 8 plots the implied volatility surface (the market's view of the future value of volatility) against time to maturity and Moneyness (defined as $S / K$, where $S$ is the price of the stock). Again, as expected, we observe that as time to maturity decreases, the volatility surface increases, reflecting the fact that a higher level of uncertainty exists regarding whether or not the option will be exercised.

\section{Risk Budgets}

Risk budgets are often used to allocate the risk of a portfolio by decomposing the total portfolio risk into the risk contribution of each component position. Portfolio standard deviation (Std), Valueat-Risk (VaR), and expected tail loss (ETL) budgets are the most popular strategies used to better understand the center-risk and tail-risk contributions. Chow and Kritzman (2001), Litterman (1996), Maillard et al. (2010), and Peterson and Boudt (2008) studied the use of portfolio Std and VaR in risk budgeting. Boudt et al. (2013) reviewed the ETL budgets. Here, we use the Std and ETL risk budgets as an investment strategy under the condition of an equal-weights portfolio. The equal-weights portfolio is widespread in practice because it does not require information on the risk and return, and supposedly provides a diversified portfolio. 


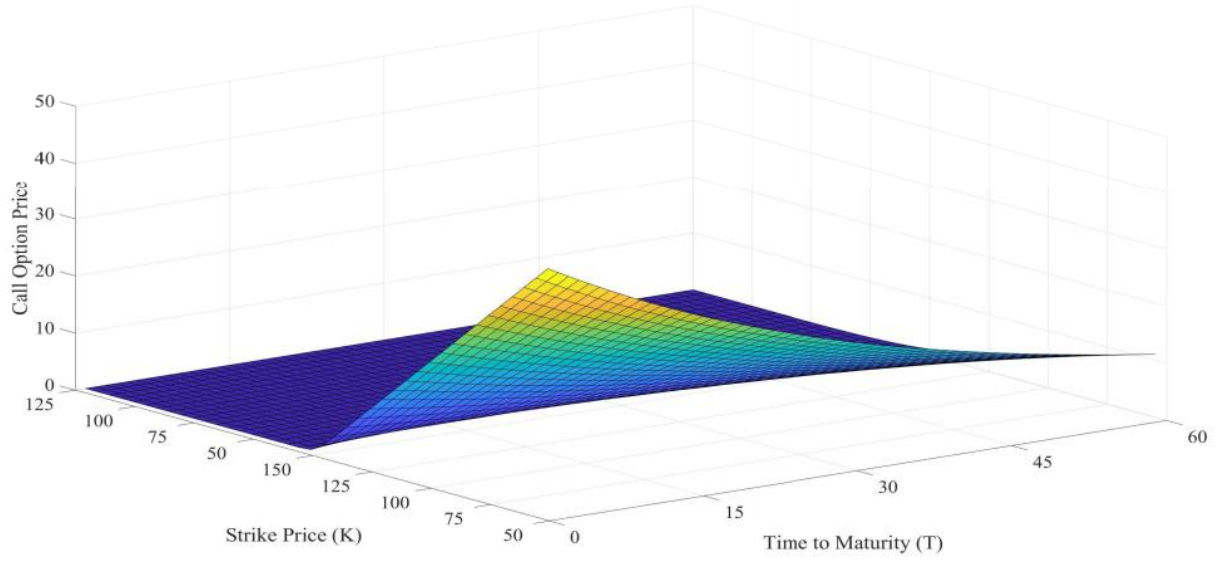

Figure 5. Call-Option prices against time to maturity and strike price

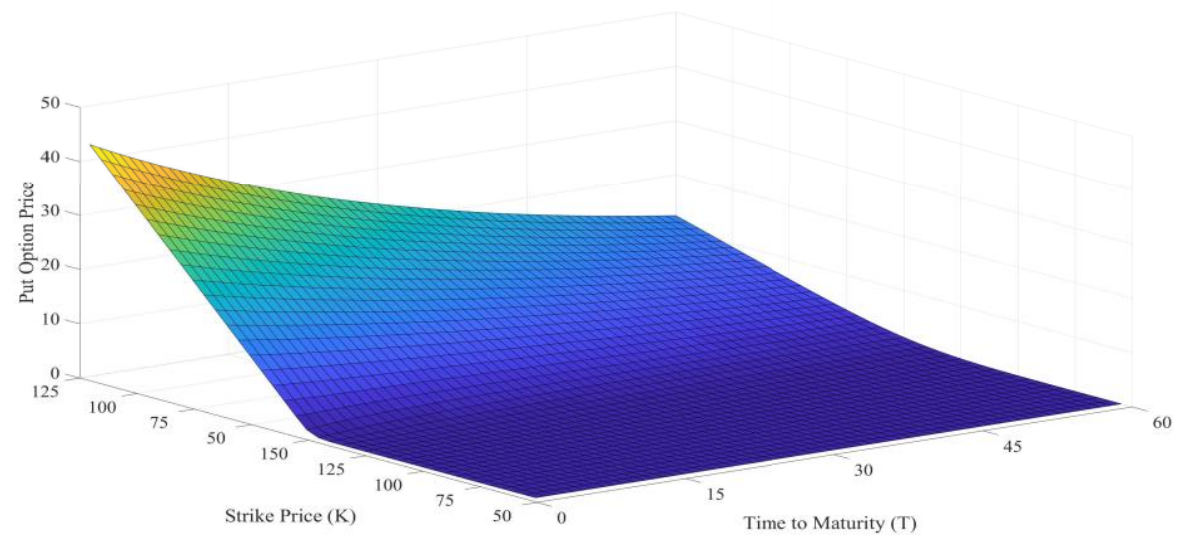

Figure 6. Put-Option prices against time to maturity and strike price

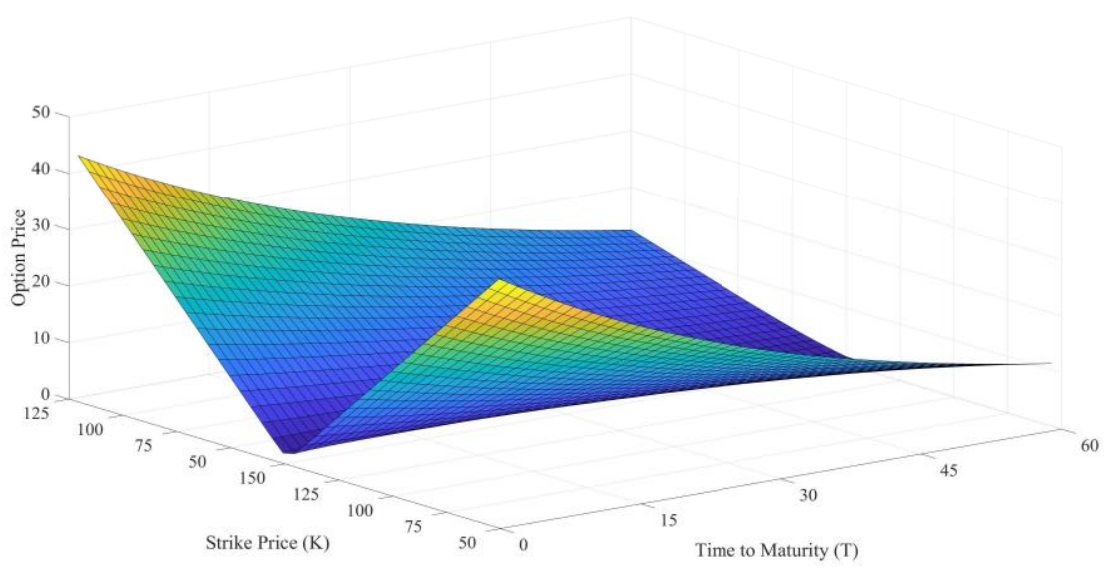

Figure 7. Call and Put-Option prices against time to maturity and strike price 


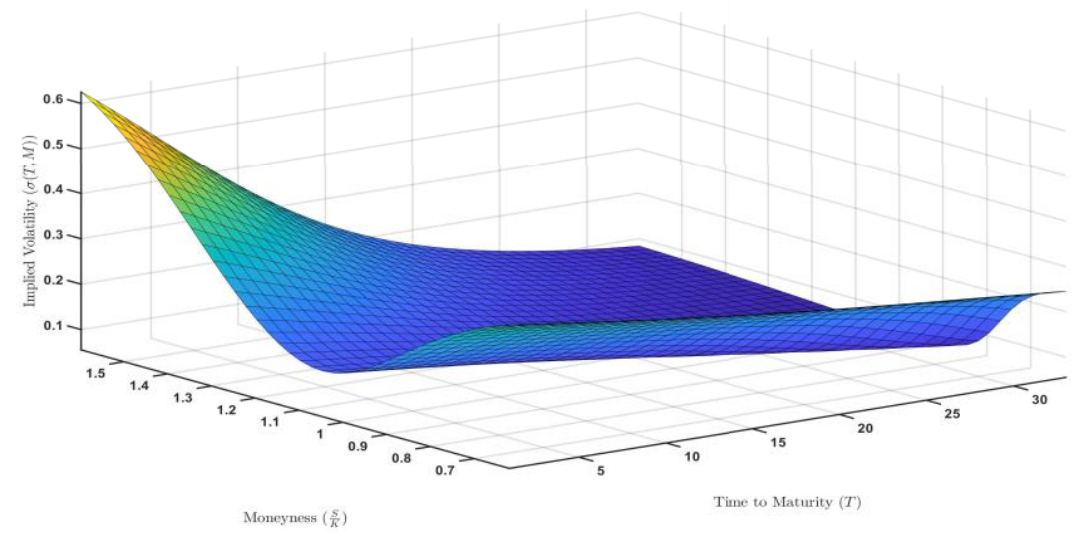

Figure 8. Implied volatility against time to maturity and Moneyness

Thus, we have the vector of portfolio weights $w=\left(w_{1}, \ldots, w_{N}\right)$ at each time point $t$, where $N=13$ and $t=$ $1,2, \ldots \ldots, 31$. We first define the marginal risk and risk contribution of the $i$-th asset in the portfolio, and then calculate the respective Std and ETL risk contributions. Let $R(w): R^{n} \rightarrow R$ denote a risk measure in the portfolio weight vector, $w$, then the marginal risk contribution of the $i$-th asset denoted by $R C_{i}$ is

$$
R C_{i}(w)=w_{i} \frac{\partial R(w)}{\partial w_{i}} .
$$

The marginal risk contribution of the $k$-th subset is

$$
R C_{M_{k}}(w)=\sum_{i \in M_{k}} R C_{i}(w)
$$

Where $M_{k} \subseteq\{1, \ldots, N\}$ for each $k=1, \ldots . ., s$, denotes $s$ subsets of portfolio assets.

Table 2 reports the estimated risk allocation of the equal-weights portfolio. It seems NegVXO has a relatively higher risk than the other factors. Meanwhile, GovTrans factor has the lowest tail risk contribution in both cases, and NegUnemploy has the lowest center risk contributions.

Thus, the tail risk diversifiers are GovTrans, DispIncome, GDP, NegInequality, NegCrimeRate, NegGenderParity, and LifeExpect. The tail risk contributors are the remaining factors. Note that NegVXO is the main risk contributor among all factors.

Table 2. Standard deviation and ETL Risk Budget

\begin{tabular}{lrrrrrr}
\hline Factors & $\begin{array}{c}\text { MCTR } \\
\text { ETL (95) }\end{array}$ & $\begin{array}{c}\text { PCTR } \\
\text { ETL (95) }\end{array}$ & $\begin{array}{c}\text { MCTR } \\
\text { ETL (99) }\end{array}$ & $\begin{array}{c}\text { PCTR } \\
\text { ETL(99) }\end{array}$ & $\begin{array}{c}\text { MCTR } \\
\text { (Std) }\end{array}$ & $\begin{array}{c}\text { PCTR } \\
\text { (Std) }\end{array}$ \\
\hline GovTrans & $-0.70 \%$ & $-7.25 \%$ & $-1.17 \%$ & $-9.38 \%$ & $3.13 \%$ & $4.75 \%$ \\
DispIncome & $-0.10 \%$ & $-1.07 \%$ & $-0.03 \%$ & $-0.27 \%$ & $-0.12 \%$ & $-0.19 \%$ \\
GDP & $-0.08 \%$ & $-0.83 \%$ & $-0.07 \%$ & $-0.59 \%$ & $0.22 \%$ & $0.33 \%$ \\
NegInequality & $-0.06 \%$ & $-0.62 \%$ & $-0.11 \%$ & $-0.87 \%$ & $-0.24 \%$ & $-0.36 \%$ \\
NegCrime & $-0.03 \%$ & $-0.31 \%$ & $0.04 \%$ & $0.32 \%$ & $0.09 \%$ & $0.13 \%$ \\
NegGenderParity & $-0.03 \%$ & $-0.26 \%$ & $-0.02 \%$ & $-0.19 \%$ & $-0.24 \%$ & $-0.37 \%$ \\
LifeExpect & $-0.02 \%$ & $-0.24 \%$ & $-0.03 \%$ & $-0.23 \%$ & $-0.02 \%$ & $-0.03 \%$ \\
Confidence & $0.13 \%$ & $1.36 \%$ & $0.18 \%$ & $1.44 \%$ & $0.73 \%$ & $1.11 \%$ \\
NegCPI & $0.32 \%$ & $3.26 \%$ & $0.38 \%$ & $3.08 \%$ & $0.19 \%$ & $0.29 \%$ \\
NegUnemploy & $0.60 \%$ & $6.14 \%$ & $1.02 \%$ & $8.18 \%$ & $-0.31 \%$ & $-0.46 \%$ \\
Sentiment & $1.33 \%$ & $13.69 \%$ & $1.78 \%$ & $14.27 \%$ & $7.93 \%$ & $12.04 \%$ \\
NegUncertainty & $3.19 \%$ & $32.84 \%$ & $4.01 \%$ & $32.16 \%$ & $19.87 \%$ & $30.18 \%$ \\
NegVXO & $5.17 \%$ & $53.30 \%$ & $6.50 \%$ & $52.09 \%$ & $34.63 \%$ & $52.58 \%$ \\
\hline
\end{tabular}




\section{Stress-Testing}

Asset and investment management firms commonly use stress-testing to determine the resilience of a given portfolio against possible undesirable financial situations (assess the risk), and then set in place any hedging strategies necessary to mitigate against possible losses. The intent is to evaluate how well the assets might weather certain market occurrences and external events. The determination of appropriate factors that may contribute to these "stressful" events, is in itself a difficult task.

In this section we consider stress testing the Index with the contemporaneously observed factors trade balance (Trade) and legal immigration (Immig), acting as stressors. The data on Trade represents the difference between the total value of US export and import of goods and services, based on the Balance of Payments. The annual data is obtained from the US Census Bureau. Immig is the total number of persons obtaining lawful permanent resident status, and is retrieved from the Department of Homeland Security. We choose these two variables as stress factors because they may be sensitive to policy makers' decisions, but they should be a priori neutral in that changes in their values do not straightforwardly imply a cause-effect relationship with the well-being of US citizens.
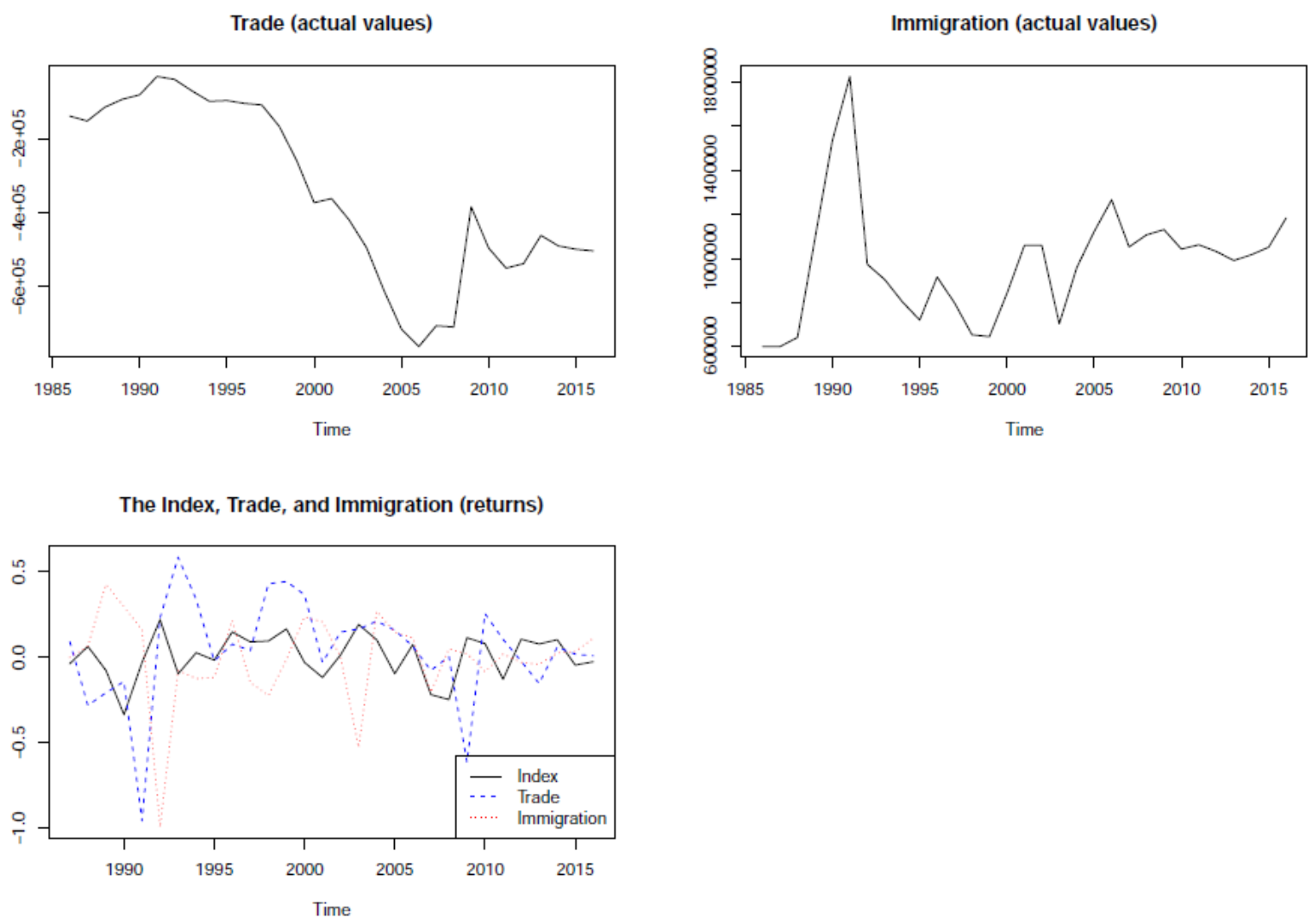

Figure 9. Time Series Plots of the Index and stress factors Trade and Immig.

The top panels of Figure 9 plots the actual values of the stress factor time series, while the bottom panel shows the log-returns of these as well as the Index, which are all white noise, as expected. In fact, a Ljung-Box test on all 3 series of returns does not detect any serial correlation, or even dependence (all p-values larger than 0.5 ). Thus we opted not to put the series through the ARMA $(1,1)-\operatorname{GARCH}(1,1)$ filter for this analysis, i.e., the ARIMA $(0,0,0)$ with zero mean suggested by AIC and BIC in Section 4 was fitted to the returns of all three series: Index, Trade, and Immig.

Starting from these three plausibly iid series, we proceeded by fitting bivariate GH models to their joint marginal distributions: Trade vs. Index, and Immig vs. Index. The models suggested by AIC and BIC had values for the tail parameter of approximately $\lambda \approx 0.4$, which is somewhat close to a VG distribution, although the latter, as well as NIG, provided substantially inferior fits. In order to compute the systemic risk measures discussed below, 10,000 simulated values were drawn from these models. Figures $10 \mathrm{a}$ and $10 \mathrm{~b}$ display the fitted contour plots from each model, overlaid with the 10,000 simulated values and the 30 observed data points. As is noted in the figures, the empirical correlation coefficients based on the observed data, suggest a weak positive relationship between Trade and the Index $(\hat{\rho}=0.15)$, 
and a moderate negative association between Immig and the Index $(\hat{\rho}=-0.44)$.

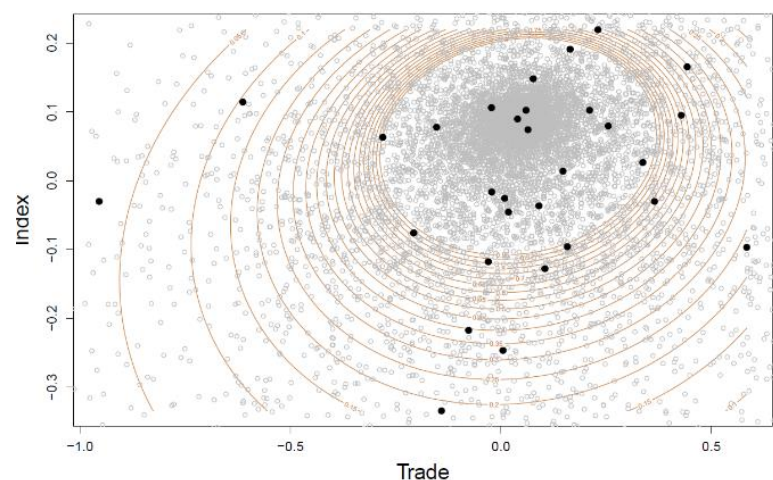

(a) Trade vs. Index $(\hat{\rho}=0.15)$.

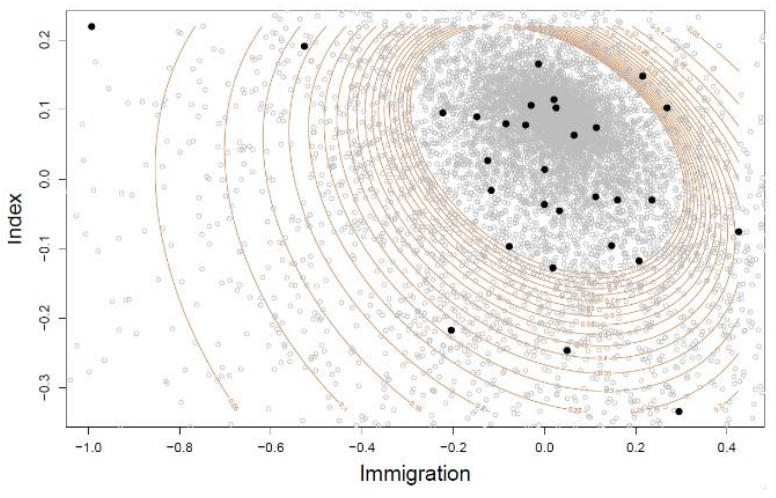

(b) Immig vs. Index $(\hat{\rho}=-0.44)$.

Figure 10. Contour plots of fitted bivariate GH models to the joint log-returns of Trade and Index (left panel), and Immig and Index (right panel). The observed data and the 10,000 simulated values from the fitted model are displayed as black and grey dots, respectively.

The assessment of how extremely negative events on the stress factors, i.e., values on their left tail, impact the Index, can be done with the various measures of systemic risk currently in vogue, all of which target the left tail of the conditional distribution of the random variable of interest, $Y$, given that the stressor, $X$, is at or below some low quantile. This notion of Conditional Value-at-Risk (CoVaR), was originally introduced by Adrian and Brunnermeier (2016).

Here, and due to the better properties pointed out by Mainik and Schaanning (2014), we opt to take the variant of CoVaR developed by Girardi and Ergun (2013). If $F_{Y \mid X}$ denotes the conditional distribution of $Y$ given $X$, each with respective distribution functions $F_{Y}$ and $F_{X}$, then we denote by $\xi_{q}$ the CoVaR at level $q$, or $\mathrm{CoVaR}_{q}$, which is defined to be

$$
\xi_{q}=\operatorname{CoVaR}_{q}=F_{Y \mid X \leq F_{X}^{-1}(q)}^{-1}(q)=\operatorname{VaR}_{q}\left(Y \mid X \leq \operatorname{VaR}_{q}(X)\right)
$$

where, using fairly standard notation from the risk modeling literature, $\operatorname{VaR}_{q}(X)=F_{X}^{-1}(q)$ denotes the $\mathrm{VaR}$ of $X$ at level $q$, which is simply the $q$-quantile of $X$. In line with this concept, the analogous value for the closely associated ES, defined as the tail mean beyond VaR, is given by (Mainik and Schaanning, 2014)

$$
\operatorname{CoES}_{q}=E\left(Y \mid Y \leq \xi_{q}, X \leq \operatorname{VaR}_{q}(X)\right) .
$$

A variation on this proposed by Biglova et al. (2014) is

$$
\operatorname{CoETL}_{q}=E\left(Y \mid Y \leq \operatorname{VaR}_{q}(Y), X \leq \operatorname{VaR}_{q}(X)\right) .
$$

The stress-testing results are presented in Table 3. The general pattern seems to be that for the same level, stress on Trade appears to have a marginally larger impact on the Index than does stress on Immig; a finding which is also consistent with the sign of the correlation coefficients noted above. However, at the highest stress level of $1 \%$, the results are mixed. 
Table 3. Left-tail systemic risk measures on the Index at different levels, based on stressing the factors Trade and Immig.

\begin{tabular}{cc|ccc}
\hline \multirow{2}{*}{ Stress } & Stress & \multicolumn{3}{|c}{ Risk Measure on Index (left tail) } \\
\cline { 3 - 5 } Factor & Level & CoES & CoVaR & CoETL \\
\hline \multirow{3}{*}{ Trade } & $10 \%$ & -0.8403 & -0.5448 & -0.4980 \\
& $5 \%$ & -1.2377 & -0.8414 & -0.6668 \\
& $1 \%$ & -1.6367 & -1.2331 & -0.9728 \\
\hline \multirow{3}{*}{ Immig } & $10 \%$ & -0.7027 & -0.4370 & -0.4748 \\
& $5 \%$ & -0.9902 & -0.7288 & -0.6645 \\
& $1 \%$ & -1.3788 & -1.2682 & -1.0084 \\
\hline
\end{tabular}

\section{Conclusion and Discussion}

We proposed an annual well-being index (SWBI) constructed as the log-returns of an equally weighted linear combination of several socioeconomic factors, in order to dynamically measure the mood of US citizens. The data, publicly available from reliable government sources, spans the 30-year period from 1986 to 2016. Although the SWBI exhibits no apparent serial dependence, we fitted an ARMA-GARCH time series model in order to capture the marginal (or cross-sectional) distribution which was consistent with a member of the generalized hyperbolic family, as predicted by contemporary best-practices financial theory (Massing, 2019). This procedure was also a prerequisite step to generating valid option prices and performing risk budgeting for the SWBI. The resulting values reveal the relationships among time to maturity, strike price, and option price, enabling the construction and valuation of insurance-type financial instruments. For pricing deep-out-of-money and deep-in-the-money options, the standard GARCH model does not perform well. Future research may therefore investigate other types of GARCH, especially EGARCH and APARCH, as these models have the capability to capture asymmetric effects on the conditional volatility.

To complete the rational finance-based valuation, we performed risk budgeting for an equally weighted portfolio, and stress-tested the index by examining the effect of the exogenous but politically-sensitive variables, trade imbalance and amount of legal immigration, on the SWBI systemic risk. Among the component series of the SWBI, the VXO volatility measure of the stock market is the greatest contributor to tail risk. Among the external factors, it appears that the level of trade imbalance tends to be associated with a larger impact on negative well-being than does immigration; a conclusion that mirrors the empirical finding that trade imbalance is positively correlated with SWBI, while immigration is negatively correlated.

The main intent of the SWBI is to provide an early-warning mechanism for downturns in the mood of US citizens. Coupled with the proper valuation of SWBI financial instruments, it is hoped that this will alert investors to potential future crises assess and aid them in setting in place hedging strategies necessary to mitigate against possible losses.

\section{References}

Adrian, T., \& Brunnermeier, M. K. (2016). CoVaR. American Economic Review, 106(7), 1705-1741. https://doi.org/10.1257/aer.20120555

Baker, S. R., Bloom, N., \& Davis, S. J. (2016). Measuring economic policy uncertainty. The Quarterly Journal of Economics, 131(4), 1593-1636. https://doi.org/10.1093/qje/qjw024

Barndorff-Nielsen, O. (1977). Exponentially decreasing distributions for the logarithm of particle size. Proceedings of The Royal Society A: Mathematical, Physical and Engineering Sciences, 353(1674), 401-419. https://doi.org/10.1098/rspa.1977.0041

Barndorff-Nielsen, O. (1997). Processes of normal inverse gaussian type. Finance and Stochastics. https://doi.org/10.1007/s007800050032

Barndorff-Nielsen, O. (2007). Normal inverse gaussian distributions and stochastic volatility modelling. Scandinavian Journal of Statistics, 24, 1-13. https://doi.org/10.1111/1467-9469.00045

Biglova, A., Ortobelli, S., \& Fabozzi, F. (2014). Portfolio selection in the presence of systemic risk. Journal of Asset Management, 15, 285-299. https://doi.org/10.1057/jam.2014.30

Blaesild, P. (1981). The two-dimensional hyperbolic distribution and related distributions with an application to johannsens bean data. Mathematical Finance, 68, 251-263. https://doi.org/10.1093/biomet/68.1.251

Blanchflower, D. D., \& Oswald, A. J. (2004). Well-being over time in Britain and the USA. Journal of Public Economics, 88(7-8), 1359-1386. https://doi.org/10.1016/S0047-2727(02)00168-8 
Boudt, K., Carl, P., \& Peterson, B. G. (2013). Asset allocation with conditional value-at-risk budgets. Journal of Risk, 15(3), 39-68. https://doi.org/10.21314/JOR.2013.258

Chorro, C. (2012). Option pricing for GARCH-type models with generalized hyperbolic innovations. Quantitative Finance, 12(7), 1079-1094. https://doi.org/10.1080/14697688.2010.493180

Chow, G., \& Kritzman, M. (2001). Risk budgets. Journal of Portfolio Management, 56-60. https://doi.org/10.3905/jpm.2001.319792

Di Tella, R., MacCulloch, R. J., \& Oswald, A. J. (2003). The macroeconomics of happiness. Review of Economics and Statistics, 85(4), 809-827. https://doi.org/10.1162/003465303772815745

Duan, J. (1995). The garch option pricing model. Mathematical Finance, 5, 13-32. https://doi.org/10.1111/j.1467-9965.1995.tb00099.x

Duffie, D. (2001). Dynamic Asset Pricing Theory( 3rd ed.). Princeton University Press, Princeton.

Ferrer-i Carbonell, A., \& Frijters, P. (2004). How important is methodology for the estimates of the determinants of happiness? The Economic Journal, 114(497), 641-659. https://doi.org/10.1111/j.1468-0297.2004.00235.x

Gerber, H. U., \& Shiu, E. S. W. (1994). Option pricing by Escher transforms. Transactions of the Society of Actuaries, 46, 99-191.

Girardi, G., \& Ergun, A. T. (2013). Systemic risk measurement: Multivariate GARCH estimation of coVaR. Journal of Banking \& Finance, 37(8), 3169-3180. https://doi.org/10.1016/j.jbankfin.2013.02.027

Johansen, S. (1988). Statistical analysis of cointegration vectors. Journal of economic dynamics and control, 12(2-3), 231-254. https://doi.org/10.1016/0165-1889(88)90041-3

Jorgensen, B. (1982). Statistical properties of the generalized inverse Gaussian distribution. volume 9 of Lecture Notes in Statistics. Springer-Verlag. https://doi.org/10.1007/978-1-4612-5698-4

Krueger, A. B. (2009). Measuring the Subjective Well-Being of Nations (1st ed.). The University of Chicago Press. https://doi.org/10.7208/chicago/9780226454573.001.0001

Litterman, R. B. (1996). Hot spots and hedges. Journal of Portfolio Management, 52-75. https://doi.org/10.3905/jpm.1996.052

Luethi, D., \& Breymann, W. (2016). ghyp: A Package on Generalized Hyperbolic Distribution and Its Special Cases. R package version 1.5.7.

Maillard, S., Roncalli, T., \& Teiletche, J. (2010). On the properties of equally weighted risk contributions portfolios. Journal of Portfolio Management, 52-75. https://doi.org/10.3905/jpm.2010.36.4.060

Mainik, G., \& Schaanning, E. (2014). On dependence consistency of Covar and some other systemic risk measures. Statistics \& Risk Modeling, 31(1), 49-77. https://doi.org/10.1515/strm-2013-1164

Massing, T. (2019). What is the best Levy model for stock indices? a comparative study with a view to time consistency. Financial Markets and Portfolio Management, 33(3), 277-344. https://doi.org/10.1007/s11408-019-00335-2

McLean, D. (2014). National and international indices of well-being: A critical analysis. Journal of the Indiana Academy of the Social Sciences, 17(1), 5.

Paolella, M. S. (2007). Intermediate Probability: A Computational Approach. John Wiley \& Sons. https://doi.org/10.1002/9780470035061

Peterson, B., \& Boudt, K. (2008). Component var for a non-normal world. Journal of Risk, 78-81.

Pflug, G., \& Werner, R. (2007). Modeling, measuring and managing risk. World Scientific. https://doi.org/10.1142/6478

Schmidt, T. (2007). Coping with copulas. In Rank, J., editor, Copulas: From Theory to Applications in Finance, pp. 3-34. Risk Books.

\section{Copyrights}

Copyright for this article is retained by the author(s), with first publication rights granted to the journal.

This is an open-access article distributed under the terms and conditions of the Creative Commons Attribution license which permits unrestricted use, distribution, and reproduction in any medium, provided the original work is properly cited. 